\title{
GEOMETRIC SEPARATION AND EXACT SOLUTIONS FOR THE PARAMETERIZED INDEPENDENT SET PROBLEM ON DISK GRAPHS
}

\section{(EXTENDED ABSTRACT)}

\author{
Jochen Alber* \\ Universität Tübingen, Wilhelm-Schickard-Institut für Informatik, \\ Sand 13, D-72076 Tübingen, Germany. \\ alber@informatik.uni-tuebingen.de
}

\author{
Jiří Fiala ${ }^{\dagger}$ \\ Charles University, KAM, DIMATIA and ITI ${ }^{\ddagger}$, Faculty of Mathematics and Physics, \\ Malostranské nám. 2/25, 11800 Prague, Czech Republic. \\ fiala@kam.mff.cuni.cz
}

\begin{abstract}
We consider the parameterized problem, whether a given set of $n$ disks (of bounded radius) in the Euclidean plane contains $k$ non-intersecting disks. We expose an algorithm running in time $n^{O(\sqrt{k})}$, that is-to our knowledge - the first algorithm for this problem with running time bounded by an exponential with a sublinear exponent.

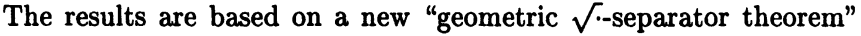
which holds for all disk graphs of bounded radius. The presented algorithm then performs, in a first step, a "geometric problem kernelization" and, in a second step, uses divide-and-conquer based on our geometric separator theorem.
\end{abstract}

Keywords: Disk graphs, independent set, fixed parameter tractable problems.

\footnotetext{
*Supported by the Deutsche Forschungsgemeinschaft (DFG), research project PEAL (Parameterized complexity and Exact Algorithms), NI 369/1-1, 1-2.

${ }^{\dagger}$ Research of this author supported in part by Czech research grant GAČR 201/99/0242 and by NATO science fellowship, administered through Norwegian Research Council project no. 143192/410 during his postdoctoral fellowship at the University of Bergen.

¥Supported by the Ministry of Education of the Czech Republic as project LN00A056.
} 


\section{Introduction}

The problem and its motivation. In this paper, we study the parameterized INDEPENDENT SET problem on disk graphs, which takes as an input a set $\mathcal{D}$ of disks in the plane and an integer $k$ and the task is to determine whether there are $k$ mutually disjoint disks in $\mathcal{D}$. The problem is motivated by various applications, among which we want to mention the area of frequency assignment problems in cellular networks.

Previous work. It is known [4] that the problem is $N P$-hard even for unit disk graphs. A way to cope with this hardness was proposed by approximation theory $[8,10]$. Very recently, Erlebach et al. [8] gave a PTAS for INDEPENDENT SET on disk graphs. In this paper, however, we are interested in exact solutions for the given problem. We want to briefly summarize various results on the parameterized and classical complexity of the INDEPENDENT SET problem for general graphs and for planar graphs (the latter are equivalent to the class of coin graphs [12], i.e., disk graphs where disks are not allowed to overlap).

In parameterized complexity theory, it is known [5] that INDEPENDENT SET is complete for the class $W[1]$, which captures intractable parameterized problems (see [6] for details). However, restricted to planar graphs, INDEPENDENT SET is fixed parameter tractable, i.e., in the class FPT (see [6] for a precise definition). For the (asymptotically) best known algorithm we get running time $O\left(c^{\sqrt{k}}+n\right)$ being sublinear in $k$ (see [2]). Moreover, very recently, Cai and Juedes [3] showed that this is best possible, in the sense that a running time of the form $O\left(c^{o(\sqrt{k})} n^{O(1)}\right)$ cannot be achieved unless 3SATE DTIME(2 $\left(2^{o(n)}\right)$, which is considered to be very unlikely.

In the classical (one-dimensional) complexity study, the best known algorithm running in time $2^{e(n)}$ with $e(n)=0.276 n$ is due to Robson [15]. Moreover, $e(n) \in o(n)$ is impossible unless 3SAT $\in D T I M E\left(2^{o(n)}\right)$ (see [11]). If restricted to planar graphs, Lipton and Tarjan applied their well-known planar separator theorem [13] to get an algorithm with $e(n)=O(\sqrt{n})$. This is the best possible asymptotic behavior for $e$ one can hope for, since otherwise an algorithm with $e(n) \in o(\sqrt{n})$ in combination with a known linear problem kernel would lead to an algorithm for the parameterized problem better than the relative lower bound shown by Cai and Juedes.

Main results and methods used. In this paper, for the case of disk graphs, we pursue the strategy of combining a geometric version of reduction to problem kernel with a divide-and-conquer approach based on an appropriate separator theorem. However, for disk graphs, so far such separator theorems are known only for so-called intersection graphs of $\tau$-neighborhood systems [7, 14], which are closely related to (unit) disk graphs with $\lambda$-precision, where all centers are at mutual distance of at least $\lambda>0$. With respect to general unit disk graphs, we quote from the introduction of Hunt et al. [10]:

"The [...] drawback is that problems such as maximum independent set [...] cannot be solved at all by the separator approach. This is because an arbitrary (unit) disk graph of n vertices can have a clique of size n." 
Table 1. Relating our results on INDEPENDENT SET on disk graphs to known results for general graphs and for planar graphs. (Lower bounds are under the assumption that 3SAT $\notin D T I M E\left(2^{o(n)}\right)$.)

\begin{tabular}{|c|c|c|}
\hline graph class & (classical) complexity & parameterized complexity \\
\hline general graphs & $\begin{array}{c}2^{0.276 n}[15] \\
\text { lower bound: } 2^{\Omega(n)}[11]\end{array}$ & W[1]-complete [5] \\
\hline disk graphs $D G_{\sigma}$ & $\begin{array}{l}2^{O(\sqrt{n} \log (n))} \\
{[\text { Rem.16] }}\end{array}$ & $\begin{array}{l}2^{O(\sqrt{k} \log (n))}[\text { Thm.15], } \\
\text { open: FPT or W[1]-h? }\end{array}$ \\
\hline $\begin{array}{l}\text { disk graphs } D G_{\sigma, \lambda} \\
\text { (with } \lambda \text {-precision) }\end{array}$ & $\begin{array}{c}2^{O(\sqrt{n})} \\
{[\text { Rem.12] }}\end{array}$ & $\begin{array}{c}O\left(2^{O(\sqrt{k} \log (k))}+n\right)[\text { Cor.17] } \\
\text { hence: FPT }\end{array}$ \\
\hline planar & $\begin{array}{c}2^{O(\sqrt{n})}[13] \\
\text { lower bound: } 2^{\Omega(\sqrt{n})}[3]\end{array}$ & $\begin{array}{l}O\left(2^{O(\sqrt{k})}+n\right)[2] \\
\text { hence: FPT }\end{array}$ \\
\hline
\end{tabular}

The key result in this paper is to show a way out of this dilemma by proving a new type of "geometric separator theorem" which holds even for the more general class of disk graphs with bounded radius ratio. Our geometric separator theorem can be seen as a generalization of (classical) separator theorems, where the guarantee is not on the size of the separator in terms of its number of vertices, but in terms of the space occupied by its disks.

This result is used to optimally solve the parameterized INDEPENDENT SET problem on disk graphs of bounded radius ratio in time $n^{O(\sqrt{k})}$ which is-to our knowledge- the first algorithm for this problem with running time bounded by a function with an exponent sublinear in $k$. In the worst case (i.e., when $k=n)$ this turns into an algorithm of running time $2^{e(n)}$ with the sublinear term $e(n)=\sqrt{n} \log (n)$; a running time which cannot be achieved for general graphs (unless 3SAT $\in D T I M E\left(2^{o(n)}\right)$ ) [11].

In addition, in the case of disk graphs with $\lambda$-precision, we can show that the INDEPENDENT SET problem is in FPT. The results are summarized in Table 1.

Various proofs are omitted in this version due to space restrictions.

\section{Preliminaries and Notation}

If $\mathcal{S}=\left\{S_{1}, \ldots, S_{n}\right\}, S_{i} \subseteq \mathbb{R}^{2}$ is a collection of geometric objects, we denote by $\bigcup \mathcal{S}=\bigcup_{i=1}^{n} S_{i}$ the union of $\mathcal{S}$. For a collection $\mathcal{S}$, let $G_{\mathcal{S}}=\left(V_{\mathcal{S}}, E_{\mathcal{S}}\right)$ denote the intersection graph of $\mathcal{S}$, i.e., $V_{\mathcal{S}}=\left\{v_{1}, \ldots, v_{n}\right\}$ and $E_{\mathcal{S}}=\left\{\left(v_{i}, v_{j}\right) \mid\right.$ $\left.S_{i} \cap S_{j} \neq \emptyset\right\}$. The collection $\mathcal{S}$ is called the representation of $G_{\mathcal{S}}$. Moreover, for a subset $\mathcal{S}^{\prime} \subseteq \mathcal{S}$, we denote by $V_{\mathcal{S}^{\prime}} \subseteq V_{\mathcal{S}}$ the subset of vertices induced by $\mathcal{S}^{\prime}$, i.e., $V_{\mathcal{S}^{\prime}}=\left\{\bar{v}_{i} \mid S_{i} \in \mathcal{S}^{\prime}\right\}$. In this setting $G_{\mathcal{S}^{\prime}}=G_{\mathcal{S}}\left[V_{\mathcal{S}^{\prime}}\right]$ is the subgraph of $G_{\mathcal{S}}$ induced by the set of vertices $V_{\mathcal{S}^{\prime}}$. 
Disk graphs. A disk $D \subseteq \mathbb{R}^{2}$ is specified by a triple $(r, x, y) \in \mathbb{R}^{3}$, where $(x, y)$ are coordinates of the center of the disk in the Euclidean plane and $r$ is its radius. The class of disk graphs, denoted by $D G$, is the set of all graphs $G$, for which we find a collection of disks $\mathcal{D}=\left\{D_{1}, \ldots, D_{n}\right\}$ such that $G=G_{\mathcal{D}}$. Note that for a collection $\mathcal{D}$, the graph $G_{\mathcal{D}}$ has a natural embedding in the plane, where $v_{i}$ sits in the position of the center of $D_{i}$.

The class of disk graphs of bounded radius ratio $\sigma$ is the subclass $D G_{\sigma} \subset D G$ of all graphs $G \in D G$ which admit a representation $\mathcal{D}=\left\{D_{1}, \ldots, D_{n}\right\}$, such that $\left(\max _{i=1, \ldots n} r_{i}\right) /\left(\min _{i=1, \ldots n} r_{i}\right) \leq \sigma$, where $r_{i}$ denotes the radius of disk $D_{i}$. The parameter $\sigma$ is called radius ratio. By a rescaling argument, for a graph $G \in D G_{\sigma}$ with representation $\mathcal{D}$, we can always achieve, that the smallest disk in $\mathcal{D}$ has radius one and, hence, all radii being upper bounded by $\sigma$.

Finally, a collection $\mathcal{D}$ is said to have $\lambda$-precision if all centers of disks are pairwise at least $\lambda$ apart (see [10, Definition 3.2]). By a rescaling argument, all disk graphs have a representation with $\lambda$-precision, however only some graphs of $D G_{\sigma}$, allow a representation with radii in $[1, \sigma]$ and precision $\lambda$. We denote this class of graphs by $D G_{\sigma, \lambda}$.

Throughout the paper, we assume that a disk graph $G$ is given together with its representation witnessing its membership in $D G, D G_{\sigma}$, or $D G_{\sigma, \lambda}$, respectively.

Grid graphs. Fix an arbitrary constant $\delta>0$, and consider the infinite grid of span $\delta$ as the planar graph $H^{\delta}=\left(W^{\delta}, E^{\delta}\right)$ with vertices $W^{\delta}=\left\{w_{i, j} \mid i, j \in \mathbb{Z}\right\}$ and edges $E^{\delta}=\left\{\left(w_{i, j}, w_{k, l}\right)|| i-j|+| k-l \mid=1\right\}$. The canonical (straightline) embedding of $H^{\delta}$ in the plane is given by putting vertex $w_{i, j}$ at the coordinates $(i \delta, j \delta)$. The set of faces $\mathcal{F}^{\delta}$ of $H^{\delta}$ contains all closed squares $F_{i, j}^{\delta}=[i \delta,(i+1) \delta] \times[j \delta,(j+1) \delta] \subseteq \mathbb{R}^{2}$. For a grid vertex $w \in W^{\delta}$, we define the face neighborhood $\hat{N}(w):=\left\{F \in \mathcal{F}^{\delta} \mid w \in F\right\}$. Similarly for $W^{\prime} \subseteq W^{\delta}$, $\hat{N}\left(W^{\prime}\right)=\bigcup_{w \in W^{\prime}} \hat{N}(w)$.

Definition 1 For a collection of disks $\mathcal{D}=\left\{D_{1}, \ldots, D_{n}\right\}$, we define $H_{\mathcal{D}}^{\delta}$ to be the smallest subgraph of the infinite grid $H^{\delta}$ induced by a set of grid points which completely covers all disks in $\mathcal{D}$. We call $H^{\delta}$ the covering grid (of span $\delta$ ) for $\mathcal{D}$. In other words, if we define the set of faces hit by $\mathcal{D}$ as $\mathcal{F}_{\mathcal{D}}^{\delta}=\left\{F \in \mathcal{F}^{\delta} \mid\right.$ $F \cap \bigcup \mathcal{D} \neq \emptyset\}$, and if $W_{\mathcal{D}}^{\delta}$ is the set of all grid points of $\mathcal{F}_{\mathcal{D}}^{\delta}$, then the covering grid $H_{\mathcal{D}}^{\delta}$ is the subgraph of $H^{\delta}$ induced by $W_{\mathcal{D}}^{\delta}$.

An example which illustrates the construction of $H_{\mathcal{D}}^{\delta}$ is given in Figure 1.

Finally, for a collection $\mathcal{D}$ of disks and a set $S \subseteq \mathbb{R}^{2}$ (e.g., a set of grid vertices or a set of faces), we call $\mathcal{D}[S]:=\{D \in \mathcal{D} \mid D \cap S \neq \emptyset\}$ the set of disks induced by $S$.

Measures. We use the standard Lebesgue measure $\mu$ in $\mathbb{R}^{2}$ as follows: For a Lebesgue measurable set $S \subseteq \mathbb{R}^{2}, \mu(S)$ denotes the size of $S$, i.e., the space in $\mathbb{R}^{2}$ occupied by $S$. In particular, for a collection of disks $\mathcal{D}=\left\{D_{1}, \ldots, D_{n}\right\}$ let $\mu(\mathcal{D})=\mu(\bigcup \mathcal{D})$ be the space covered by the union of disks $D_{1}, \ldots, D_{n}$. 


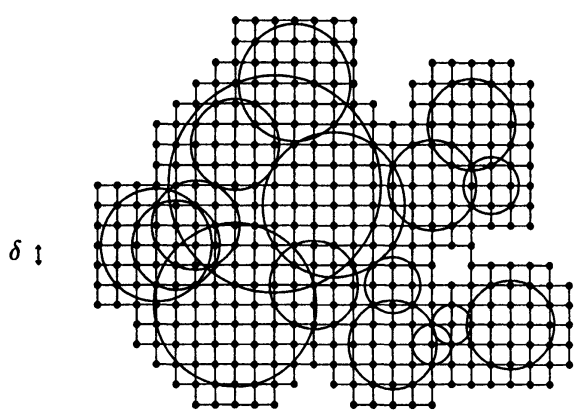

Figure 1. Constructing the grid graph $H_{\mathcal{D}}^{\delta}$ induced by a collection of disks $\mathcal{D}$.

Definition 2 Let $\mathbb{G}$ be a graph class closed under taking subgraphs. A function $\xi: \mathbb{G} \rightarrow \mathbb{R}^{+}$, that is monotonous with respect to the subgraph ordering, i.e., $\xi(G) \leq \xi\left(G^{\prime}\right)$ if $G \subseteq G^{\prime}$, and for which $\xi((\emptyset, \emptyset))=0$, is called a graph measure.

Example 3 We specify two graph measures which play a decisive role throughout the paper.

1 The usual counting measure $|\cdot|$ which assigns to any graph $G$ the size of its vertex set $\left|V_{G}\right|$ is clearly a graph measure.

2 The Lebesgue measure $\mu(\cdot)$ assigning to a disk graph $G_{\mathcal{D}}$ with representation $\mathcal{D}$ the value $\mu\left(G_{\mathcal{D}}\right)=\mu(\mathcal{D})$ is a graph measure for $D G$, when we restrict the subgraph ordering to $G_{\mathcal{D}} \subseteq G_{\mathcal{D}^{\prime}} \Leftrightarrow \mathcal{D} \subseteq \mathcal{D}^{\prime}$.

\section{A Geometric Problem Kernelization}

Reduction to problem kernel is a core technique in the design of fixed parameter algorithms.

Definition 4 Let $\mathcal{L}$ be a parameterized problem, i.e., $\mathcal{L}$ consists of pairs $(I, k)$, where problem instance $I$ has a solution of size $k$ (the parameter). Reduction to problem kernel, then, means to replace problem $(I, k)$ by a "reduced" problem $\left(I^{\prime}, k^{\prime}\right)$ (called problem kernel) such that

$$
k^{\prime} \leq c \cdot k, \quad\left|I^{\prime}\right| \leq p(k), \quad \text { and } \quad(I, k) \in \mathcal{L} \text { iff }\left(I^{\prime}, k^{\prime}\right) \in \mathcal{L},
$$

where $c$ is a constant. Furthermore, we require that the reduction from $(I, k)$ to $\left(I^{\prime}, k^{\prime}\right)$ is computable in time $T_{K}(|I|, k)$, which is a polynomial.

It is well-known that a parameterized problem is fixed parameter tractable if and only if it admits a reduction to a problem kernel.

For disk graphs, we can prove a geometric version of a problem kernel, where the size of the reduced instance is upper bounded by $O(k)$, when measured by the (Lebesgue) measure $\mu(\cdot)$ instead of the counting measure $|\cdot|$. 


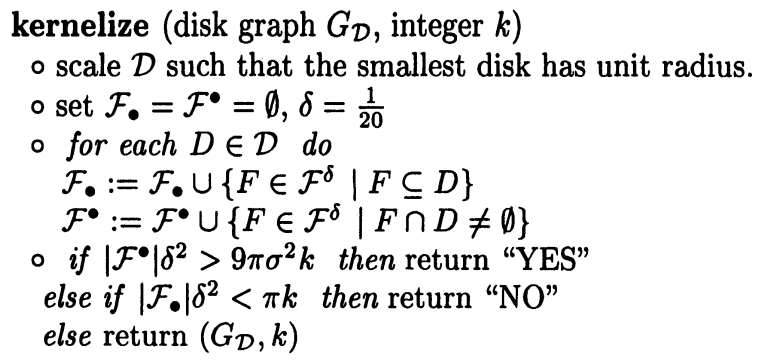

Figure 2. Geometric problem kernel reduction.

Proposition 5 For the parameterized INDEPENDENT SET (IS) problem on $D G_{\sigma}$ there exists a "geometric" problem kernel, i.e., there is a procedure, that transforms an instance $\left(G_{\mathcal{D}}, k\right)$ to an instance $\left(G_{\mathcal{D}^{\prime}}, k\right)$, such that $\left(G_{\mathcal{D}}, k\right) \in I S$ iff $\left(G_{\mathcal{D}^{\prime}}, k\right) \in I S$ and

$$
\pi k \leq \mu\left(G_{\mathcal{D}^{\prime}}\right) \leq 9 \pi \sigma^{2} k
$$

Proof. By our assumptions all disks in $\mathcal{D}$ have radius in the range $[1, \sigma]$.

Observe first, that $\mu\left(G_{\mathcal{D}}\right)>9 \pi \sigma^{2} k$ implies that $\left(G_{\mathcal{D}}, k\right) \in I S$. We use the fact that $\mu(\mathcal{D}[N(v)]) \leq(3 \sigma)^{2} \pi$ for any vertex $v \in V$, i.e., that the neighborhood of any vertex may occupy the space at most $9 \pi \sigma^{2}$. And, secondly, if $\mu\left(G_{\mathcal{D}}\right)<\pi k$, then $\left(G_{\mathcal{D}}, k\right) \notin I S$, since the representation of any independent set of $k$ vertices needs space at least $\pi k$. The procedure which in linear time transforms $\left(G_{\mathcal{D}}, k\right)$ to $\left(G_{\mathcal{D}^{\prime}}, k\right)$ with $\mu\left(G_{\mathcal{D}^{\prime}}\right) \leq 9 \pi \sigma^{2} k$ is given in Figure 2 .

Note that this is not a problem kernel according to Definition 4 , since the size of $G_{\mathcal{D}}$ is measured by the (Lebesgue) measure $\mu(\cdot)$, which, in general, is not related to the (input) size of $G$. For disk graphs with $\lambda$-precision, however, we can prove an upper bound the counting measure by the Lebesgue measure.

Lemma 6 Let $G_{\mathcal{D}}=(V, E) \in D G_{\sigma, \lambda}$ be a graph with and representation $\mathcal{D}$. Then, $|V| \leq 4 \pi^{-1} \lambda^{-2} \mu\left(G_{\mathcal{D}}\right)$.

Corollary 7 The parameterized INDEPENDENT SET problem on disk graphs $D G_{\sigma, \lambda}$ (with $\lambda$-precision) admits a linear problem kernel (in terms of the counting measure) of size $c=36\left(\frac{\sigma}{\lambda}\right)^{2} k$, which can be computed in linear time.

\section{A Geometric Separator Theorem}

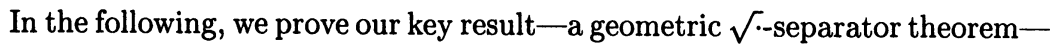
that makes our divide-and-conquer strategy work.

\subsection{Classical $\sqrt{ }$--separator theorems}

We start with a somewhat generalized notion of separator theorems. 
Definition 8 Let $G=(V, E)$ be an undirected graph. A separator $V_{S} \subseteq V$ of $G$ partitions $V$ into two parts $V_{A}$ and $V_{B}$ such that

- $V_{A} \dot{\cup} V_{S} \dot{\cup} V_{B}=V$, and

- no edge joins a vertex of $V_{A}$ to $V_{B}$.

The triple $\left(V_{A}, V_{S}, V_{B}\right)$ is also called a separation of $G$.

In order to provide a quantitative approach to separators, we need the notion of "measure" as introduced in Section 2.

Definition 9 Let $\xi$ be a graph measure. An $f(\cdot)$-separator theorem for the measure $\xi$ (and constants $\alpha<1, \beta>0$ ) on a class of graphs $\mathbb{G}$ which is closed under taking subgraphs is a theorem of the following form:

For any $G \in \mathbb{G}$ there exists a separation $\left(V_{A}, V_{S}, V_{B}\right)$ of $G$ such that

$$
\begin{aligned}
& 1 \xi\left(G\left[V_{S}\right]\right) \leq \beta \cdot f(\xi(G)) \\
& 2 \xi\left(G\left[V_{A}\right]\right), \xi\left(G\left[V_{B}\right]\right) \leq \alpha \cdot \xi(G)
\end{aligned}
$$

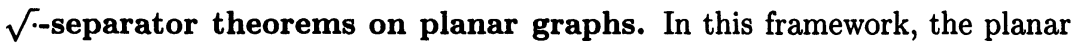
separator theorem due to Lipton and Tarjan [13] can be formulated as follows.

Theorem 10 On the class of planar graphs, there exists a $\sqrt{--s e p a r a t o r}$ theorem for the counting measure $|\cdot|$ with constants $\alpha=2 / 3$ and $\beta=2 \sqrt{2}$. Moreover, the separation can be found in linear time.

$\sqrt{ } \cdot$-separator theorems on disk graphs with $\lambda$-precision. In terms of geo-

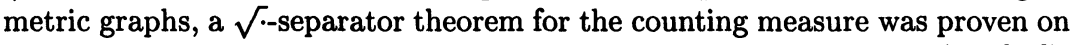
the class of intersection graphs of so-called $\tau$-neighborhood systems (see [10]). Here, a $\tau$-neighborhood system is a collection $\mathcal{B}=\left\{B_{1}, \ldots, B_{n}\right\}$ of balls in a space of arbitrary fixed dimension, such that the intersection of any $(\tau+1)$ distinct balls in $\mathcal{B}$ is empty. It can be verified that every unit disk graph with $\lambda$-precision is an intersection graph of a $\tau$-neighborhood system ( $\tau$ depending on $\lambda$ ) and, vice versa, every intersection graph of a $\tau$-neighborhood system in $\mathbb{R}^{2}$ is $\lambda$-precision disk graph ( $\lambda$ being the minimum distance between the centers of any two disks), see [10]. In the two-dimensional case the corresponding separator theorem reads as follows (see [14, Theorem 2.5] and [7, Theorem 5.1]):

Theorem 11 On the class of intersection graphs of $\tau$-neighborhood systems,

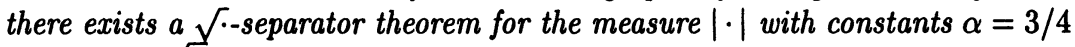
and $\beta=O(\sqrt{\lambda})$. Moreover, the separation can be found in linear time.

Remark 12 As exhibited, e.g., in [1, Section 4.1], a divide-and-conquer approach yields that INDEPENDENT SET on intersection graphs of $\tau$-neighborhood systems, and hence, on unit disk graphs with $\lambda$-precision as well as for graphs from $D G_{\sigma, \lambda}$, can be solved in time $2^{O(\sqrt{n})}$. 


\subsection{A new geometric $\sqrt{- \text {-separator theorem }}$}

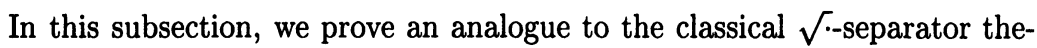
orems for the class $D G_{\sigma}$ of disk graphs with bounded radius ratio. Note that graphs in $D G_{\sigma}$ may contain arbitrary large cliques, which means that a $\sqrt{--}$ separator theorem does not hold for the counting measure. Using the Lebesgue measure instead, we get the following result.

Theorem 13 On the class $D G_{\sigma}$ of disk graphs with bounded radius ratio, there

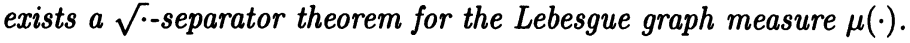

More precisely there exist constants $\alpha<1$ and $\beta$ such that, for every graph $G_{\mathcal{D}} \in D G_{\sigma}$ with representation $\mathcal{D}$, we find three sets $\mathcal{D}_{A}, \mathcal{D}_{S}, \mathcal{D}_{B} \subseteq \mathcal{D}$, such that $\left(V_{\mathcal{D}_{A}}, V_{\mathcal{D}_{S}}, V_{\mathcal{D}_{B}}\right)$ is a separation for $G_{\mathcal{D}}$, satisfying

$$
\begin{aligned}
& 1 \mu\left(\mathcal{D}_{S}\right) \leq \sigma^{2} \beta \sqrt{\mu(\mathcal{D})}, \\
& 2 \mu\left(\mathcal{D}_{A}\right), \mu\left(\mathcal{D}_{B}\right) \leq \alpha \mu(\mathcal{D}) .
\end{aligned}
$$

Moreover, this separation can be found in time linear in $|\mathcal{D}|$.

The idea for the proof of Theorem 13 is to construct the covering grid $H_{\mathcal{D}}^{\delta}$ (of suitable span) for a given collection $\mathcal{D}$ of disks. Then, in a second step, one

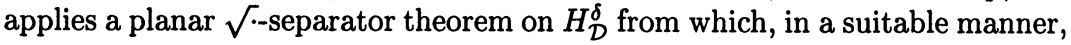
the three sets $\mathcal{D}_{A}, \mathcal{D}_{S}, \mathcal{D}_{B} \subseteq \mathcal{D}$ will be constructed. We need the following key result interrelating the space covered by $\mathcal{D}$ with the size of the covering grid.

Proposition 14 For any $\varepsilon$ there exists a $\delta$ such that for any set $\mathcal{D}$ of disks of radius at least one, we have $\left|W_{\mathcal{D}}\right| \leq(1+\varepsilon) \delta^{-2} \mu(\mathcal{D})$.

Proof. (of Theorem 13) The sets $\mathcal{D}_{S}, \mathcal{D}_{A}$ and $\mathcal{D}_{B}$ will be determined according to the procedure given in Figure 3. We now prove that, indeed $\left(V_{\mathcal{D}_{A}}, V_{\mathcal{D}_{S}}, V_{\mathcal{D}_{B}}\right)$ is a separation of $G$ and that properties (1.) and (2.) of the theorem hold for the computed sets $\mathcal{D}_{S}, \mathcal{D}_{A}$, and $\mathcal{D}_{B}$ :

$\left(V_{\mathcal{D}_{A}}, V_{\mathcal{D}_{S}}, V_{\mathcal{D}_{B}}\right)$ is a separation of $G$ : Showing that $\left(V_{\mathcal{D}_{A}}, V_{\mathcal{D}_{S}}, V_{\mathcal{D}_{B}}\right)$ is a separation of $G_{\mathcal{D}}$ is equivalent to proving that $\bigcup \mathcal{D}_{A} \cap \bigcup \mathcal{D}_{B}=\emptyset$. Recall that $\left(W_{A}, W_{S}, W_{B}\right)$ is the separation of the covering grid $H_{\mathcal{D}}^{\delta}$ obtained by the algorithm of Lipton and Tarjan. First of all we claim that

$$
\bigcup \mathcal{D}_{A} \cap W_{\mathcal{D}}^{\delta} \subseteq W_{A}, \text { and } \bigcup \mathcal{D}_{B} \cap W_{\mathcal{D}}^{\delta} \subseteq W_{B}
$$

To see this, note that $\bigcup \mathcal{D}_{A} \cap W_{S}=\emptyset$, since if there is a disk $D \in \mathcal{D}_{A}$ containing a point $w \in W_{S}$-by construction of $\mathcal{D}_{S}$-we had $D \in \mathcal{D}_{S}$. Suppose now that there is a vertex $w_{B} \in W_{B}$ which lies in a disk $D \in \mathcal{D}_{A}$. By definition of the set $\mathcal{D}_{A}$, we find a vertex $w_{A} \in W_{A}$ inside $D$ as well. Then, there exists a path $P$ in $H_{\mathcal{D}}^{\delta}$ which connects $w_{A}$ and $w_{B}$ and which is completely located inside the disk $D$. Since $\left(W_{A}, W_{S}, W_{B}\right)$ is a separation of $H_{\mathcal{D}}^{\delta}$, there exists a vertex of $W_{S}$ on $P$ contradicting $\cup \mathcal{D}_{A} \cap W_{S}=\emptyset$. This also implies that 


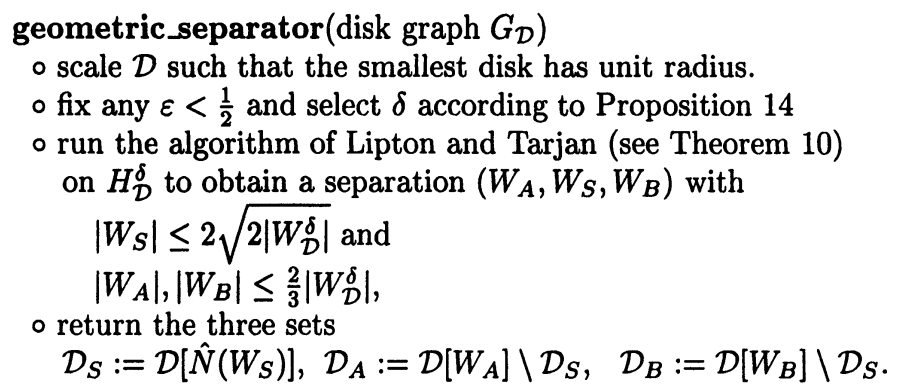

Figure 3. Separator algorithm corresponding to Theorem 13.

$\cup \mathcal{D}_{A} \cap W_{B}=\emptyset$. Since $W_{\mathcal{D}}^{\delta}=W_{A} \cup W_{S} \cup W_{B}$, we get $\bigcup \mathcal{D}_{A} \cap W_{\mathcal{D}}^{\delta} \subseteq W_{A}$. The property $\bigcup \mathcal{D}_{B} \cap W_{\mathcal{D}}^{\delta} \subseteq W_{B}$ follows similarly.

Assume now, for contradiction that vertices $v_{A} \in V_{\mathcal{D}_{A}}$ and $v_{B} \in V_{\mathcal{D}_{B}}$ form an edge of $E_{\mathcal{D}}$, that means there exists some point $z$ in $D_{A} \cap D_{B}$. Let $F_{z} \in \mathcal{F}^{\delta}$ be any of the (at most four) squares containing $z$, and let $W_{z}$ be the four grid vertices adjacent to $F_{z}$ in $H_{\mathcal{D}}^{\delta}$.

We first consider the case when $D_{B}$ does not intersect $W_{z}$. Then it intersects one side of $F_{z}$, and since $\delta \ll 1$ it contains two grid points of the square sharing this side. Note that we use equation (2) in the sense that all grid points intersected by $D_{B}$ belong to $W_{B}$. Then as $D_{A}$ must contain at least one point of $W_{z}$ (if not, either there is no possible place for $z$ or $W_{A} \cap W_{B} \neq \emptyset$ ).

In this case, or when we symmetrically exchange subscripts $A$ and $B$, and also when both $D_{A}$ and $D_{B}$ intersect $W_{z}$, there are two grid points $w_{A} \in D_{A}$, $w_{B} \in D_{B}$, that are in $H_{\mathcal{D}}^{\delta}$ at distance at most two. Since $w_{A}$ and $w_{B}$ must be separated by $W_{S}$, and at the same time they belong to $\hat{N}\left(W_{S}\right)$, that is in contradiction with the definition of sets $\mathcal{D}_{A}$ and $\mathcal{D}_{B}$.

ad property (1.): Consider a vertex $w \in W_{S}$ and the neighborhood $\hat{N}(w)$. All disks in $\mathcal{D}$ which intersect $\hat{N}(w)$ must lie inside a cycle of radius $(2 \sigma+\sqrt{2} \delta)$ centered at $w$. This is clear, since disks in $\mathcal{D}$ have radius bounded by $\sigma$ and since the grid has span $\delta$. More formally, we get

$$
\mu(\mathcal{D}[\hat{N}(w)]) \leq(2 \sigma+\sqrt{2} \delta)^{2} \pi .
$$

Moreover, this implies that

$$
\begin{aligned}
\mu\left(\mathcal{D}_{S}\right) & =\mu\left(\mathcal{D}\left[\hat{N}\left(W_{S}\right)\right]\right)=\mu\left(\bigcup_{w \in W_{S}} \mathcal{D}[\hat{N}(w)]\right) \\
& \leq \sum_{w \in W_{S}} \mu(\mathcal{D}[\hat{N}(w)]) \leq\left((2 \sigma+\sqrt{2} \delta)^{2} \pi\right)\left|W_{S}\right| \leq 5 \sigma^{2} \pi\left|W_{S}\right|
\end{aligned}
$$

Since, by our choice, $\varepsilon<\frac{1}{2}$ we may use in the last step $\sqrt{2} \delta<\frac{1}{6} \leq \frac{\sigma}{6}$. Using property (a) of step (3.) of the algorithm in Figure 3 and Proposition 14, we 
have $\left|W_{S}\right| \leq \beta^{\prime} \sqrt{\left|W_{\mathcal{D}}\right|} \leq \beta^{\prime} \sqrt{\frac{1+\varepsilon}{\delta^{2}} \mu(\mathcal{D})}$ which together with the estimate (3) establishes

$$
\mu\left(\mathcal{D}_{S}\right) \leq \sigma^{2} \beta \sqrt{\mu(\mathcal{D})} \text { for } \beta=\frac{5 \pi \beta^{\prime}}{\delta} \sqrt{(1+\varepsilon)}
$$

ad property (2.): First of all, observe that the set $\bigcup \mathcal{D}_{A}$ is completely covered by the square faces of the subgraph $H_{\mathcal{D}}^{\delta}\left[W_{A}\right]$, induced by the vertices of $W_{A}$. To see this, suppose there is a point $z \in D$ (for some $D \in \mathcal{D}_{A}$ ) which lies in some square $F_{z} \in \mathcal{F}^{\delta}$ of $H_{\mathcal{D}}^{\delta}$ but not of $H_{\mathcal{D}}^{\delta}\left[W_{A}\right]$. As above, if the four vertices adjacent to $F_{z}$ host a vertex of $W_{B}$ or $W_{S}$, we get $D \cap \hat{N}\left(W_{S}\right) \neq \emptyset$, a contradiction. By this observation and by the fact that $\left|W_{A}\right| \leq \alpha^{\prime}\left|W_{\mathcal{D}}\right|$, we get

$$
\mu\left(\mathcal{D}_{A}\right) \leq \mu\left(H_{\mathcal{D}}^{\delta}\left[W_{A}\right]\right) \leq \delta^{2}\left|W_{A}\right| \leq \delta^{2} \alpha^{\prime}\left|W_{\mathcal{D}}^{\delta}\right| \leq \alpha^{\prime}(1+\varepsilon) \mu(\mathcal{D}),
$$

where Proposition 14 was used in the last step.

Similarly, one proves $\mu\left(\mathcal{D}_{B}\right) \leq \alpha^{\prime}(1+\varepsilon) \mu(\mathcal{D})$.

We note here that by our choice of $\varepsilon=\frac{1}{4}$, we get $\alpha=\alpha^{\prime}(1+\varepsilon)=\frac{5}{6}$; but $\alpha$ can be arbitrarily close to $\alpha^{\prime}$ by a sufficiently small choice of $\varepsilon$. Then, however, we have to consider the tradeoff of getting a small $\beta$ according to Equation (4) on the one hand, and enlarging $\left|W_{\mathcal{D}}^{\delta}\right|$ on the other hand.

\section{The Algorithm and its Analysis}

We use the geometric kernelization of Section 3 and a divide-and-conquer approach based on the geometric separator theorem from Section 4.2 to derive an algorithm for the INDEPENDENT SET problem on disk graphs $D G_{\sigma}$.

Theorem 15 Let $\mathcal{D}$ be a collection of $n$ disks with $G_{\mathcal{D}} \in D G_{\sigma}$. Then, there is an algorithm running in time $n^{O(\sqrt{k})}$ which decides if $G_{\mathcal{D}}$ admits an independent set of size at least $k$, and if so constructs one.

Proof. On input instance $\left(G_{\mathcal{D}}, k\right)$, in a first step, perform the geometric kernelization explained in Section 3. After this step, without loss of generality, we may assume that $\mu(\mathcal{D}) \leq c k$ for the constant $c$ given in Proposition 5. In a second step, the divide-and-conquer procedure indep_set shown in Figure 4 is applied to the instance $(\mathcal{D}, c k)$.

Denote by $T(n, s)$ the time needed to execute $\operatorname{indep\_ set}(\mathcal{D}, s)$ on a collection of $n$ disks $\mathcal{D}$ with $\mu(\mathcal{D}) \leq s$. Let $p(|\mathcal{D}|)$ be the polynomial time needed to compute the sets $\mathcal{D}_{S}, \mathcal{D}_{A}$, and $\mathcal{D}_{B}$ according to Theorem 13 , and $q(|\mathcal{D}|)$ be the polynomial time needed to perform constructions of $\mathcal{D}_{A}^{\prime}$ and $\mathcal{D}_{B}^{\prime}$. Note that in $\mathcal{D}_{S}$ at most $\left\lfloor\frac{\beta \sqrt{s}}{\pi}\right\rfloor$ many disks can be independent, since $\mu\left(\mathcal{D}_{S}\right) \leq \beta \sqrt{s}$ and every disk has radius at least one. Hence, the total number of independent sets in $G_{\mathcal{D}_{S}}$ is upper bounded by

$$
\sum_{i=0}^{\left\lfloor\frac{\beta \sqrt{\delta}}{\pi}\right\rfloor} n() i \leq n^{\widehat{\beta} \sqrt{s}}
$$




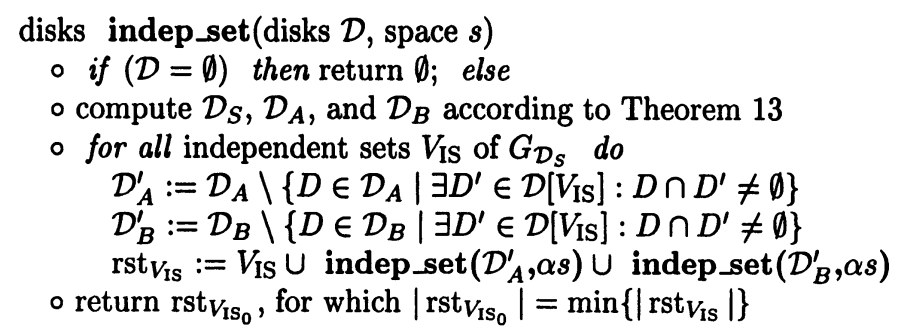

Figure 4. Divide-and-conquer algorithm for INDEPENDENT SET on disk graphs of bounded radius ratio based on our geometric separator theorem.

where $\widehat{\beta}$ is some constant. Then, the recursion we have to solve in order to compute an upper bound on $T(n, s)$ reads as follows:

$$
T(n, s) \leq p(n)+n^{\hat{\beta} \sqrt{s}} \cdot q(n) \cdot 2 T(n, \alpha s) .
$$

Hence, for $n$ large enough, and a suitable constant $\widetilde{\beta}$ we have

$$
\begin{aligned}
T(n, s) & \leq n^{\tilde{\beta} \sqrt{s}} \cdot T(n, \alpha s) \leq \prod_{i=0}^{\log _{1 / \alpha}(s)} n^{\tilde{\beta} \sqrt{\alpha^{i} s}} \cdot T(n, 1) \\
& \leq n^{\tilde{\beta} \sqrt{s}\left(\sum_{i=0}^{\infty}(\sqrt{\alpha})^{i}\right)} \cdot T(n, 1)=n^{\frac{\tilde{\beta} \sqrt{s}}{1-\sqrt{\alpha}}} \cdot T(n, 1) .
\end{aligned}
$$

Note that $T(n, 1)$ is constant, since $\mu(\mathcal{D}) \leq 1$ implies $\mathcal{D}=\emptyset$, because for every disk $D$ we have $\mu(D) \geq \pi$. By plugging in the values $n=|\mathcal{D}|$ and $s=c k$, we obtain the running time as we have claimed.

Remark 16 In the worst case $k=n$, we have an $O\left(2^{O(\sqrt{n} \log (n))}\right)$-algorithm, a running time with a sublinear exponent that cannot be achieved for general graphs (unless 3SATE DTIME $\left(2^{o(n)}\right)$ ) [11].

Corollary 17 By Corollary 7, it follows that the parameterized INDEPENDENT SET problem on disk graphs $D G_{\sigma, \lambda}$ (with $\lambda$-precision) can be solved in time $O\left(k^{O(\sqrt{k})}+n\right)$, hence, the problem is fixed parameter tractable.

We leave it as an open problem, whether INDEPENDENT SET on disk graphs is in FPT or complete for the classes $W[1]$ or $W[2]$, respectively. We want to emphasize, that we are not aware of a (non-artificial) $W[1]$-hard problem which can be solved in time bounded by an exponential with a sublinear exponent. Similarly, its W[1]-hardness would expose the first example of a fixed parameter intractable problem that simultaneously allows a PTAS.

Acknowledgments. We thank Thomas Erlebach and Rolf Niedermeier for introducing us to the problem and for fruitful discussion and valuable comments 
during this project. We thank also Jan Arne Telle for his hospitality and encouragement during our visit at University of Bergen. Finally, we thank Jiří Matoušek for pointing us to an elegant tool which was used in the proof of Lemma 6.

\section{References}

[1] Alber, J., Fernau, H., and Niedermeier., R. (2001a). Graph separators: a parameterized view. In Proc. 7th COCOON 2001, pages 318-327. Springer-Verlag LNCS 2108.

[2] Alber, J., Fernau, H., and Niedermeier., R. (2001b). Parameterized complexity: exponential speed-up for planar graph problems. In Proc. 28th ICALP 2001, pages 261-272. Springer-Verlag LNCS 2076.

[3] Cai, L. and Juedes, D. W. (2001). On the existence of subexponential parameterized algorithms. revised version of: Subexponential parameterized algorithms collapse the W-hierarchy. In Proc. 28th ICALP 2001, pages 273-284. SpringerVerlag LNCS 2076.

[4] Clark, B. N., Colbourn, C. J., and Johnson, D. S. (1990). Unit disk graphs. Discrete Math., 86(1-3):165-177.

[5] Downey, R. G. and Fellows, M. R. (1995). Fixed-parameter tractability and completeness II: On completeness for W[1]. Theor. Comput. Sci., 141:109-131.

[6] Downey, R. G. and Fellows, M. R. (1999). Parameterized Complexity. Texts and Monographs in Computer Science. Springer-Verlag.

[7] Eppstein, D., Miller, G. L., and Teng, S.-H. (1993). A deterministic linear time algorithm for geometric separators and its applications. In Proc. of 9th ACM Symposium on Computational Geometry, pages 99-108.

[8] Erlebach, T., Jansen, K., and Seidel, E. (2001). Polynomial-time approximation schemes for geometric graphs. In Proc. 12th ACM-SIAM SODA, pages 671-679.

[9] Hochbaum, D. S. and Maass, W. (1985). Approximation schemes for covering and packing problems in image processing and VLSI. Journal of the ACM, 32(1):130-136.

[10] Hunt, H. B., Marathe, M. V., Radhakrishnan, V., Ravi, S., Rosenkrantz, D. J., and Stearns, R. E. (1998). NC-approximation schemes for NP- and PSPACEhard problems for geometric graphs. Journal of Algorithms, 26(2):238-274.

[11] Impagliazzo, R., Paturi, R., and Zane, F. (1998). Which problems have strongly exponential complexity? In Proc. of the 39th IEEE FOCS, pages 653-664.

[12] Koebe, P. (1936). Kontaktprobleme der konformen Abbildung. Berichte über die Verhandlungen d. Sächs. Akad. d. Wiss. Math-Phys. Klasse, 88:141-164.

[13] Lipton, R. J. and Tarjan, R. E. (1980). Applications of a planar separator theorem. SIAM Journal on Computing, 9:615-627.

[14] Miller, G. L., Teng, S.-H., and Vavasis, S. A. (1991). A unified geometric approach to graph separators. In Proc. of the 32nd IEEE FOCS, pages 538-547.

[15] Robson, J. M. (1986). Algorithms for maximum independent sets. Journal of Algorithms, 7:425-440. 